\title{
Zwischen Overcriminalization und Overpunishment: Die US-amerikanische Mens-Rea-Debatte und die politischen Dimensionen des Schuldprinzips
}

\author{
Monika Simmler, St. Gallen
}

\section{A. Einleitung}

Dass die USA nicht nur ein „Kriminalitätsproblem“, sondern vielmehr auch ein „Strafrechtsproblem“ haben, ist in unseren Breitengraden gängiger Konsens. Die Ursachen dafür sind sicherlich vielschichtig. Die als „Expansion“ des Strafrechts wahrgenommene Problematik rund um die zehntausenden Straftatbestände ${ }^{1}$ mündete jedoch in den letzten Jahren primär in eine intensive Debatte über die sog. Overcriminalization. ${ }^{2}$ Diese „Überkriminalisierung“ nimmt dabei sowohl Politik als auch Wissenschaft in Anspruch und hat unter anderem dazu geführt, dass in der Strafrechtswissenschaft des Common Law Kriminalisierungstheorien den Straftheorien mittlerweile den Rang abgelaufen haben. ${ }^{3}$ Die Vereinigten Staaten seien „the world's largest jailer“, da sie - auch wenn sie nur ca. 5\% der Weltbevölkerung beheimaten - für rund $25 \%$ der weltweiten Gefängnispopulation verantwortlich sind. ${ }^{4}$ Obwohl damit noch keineswegs festgestellt ist, dass die Ursache für dieses Phänomen tatsächlich in einer Ausweitung des materiellen Strafrechts auf immer mehr Statutes und Re-

1 Gemäß verschiedenen Kommentatoren soll es in den USA schätzungsweise 4.500 Bundesgesetze und mehr als 300.000 Bundesverordnungen geben, welche Strafbestimmungen enthalten. Die genaue Anzahl ist jedoch sowohl aufgrund der Unübersichtlichkeit als auch aufgrund divergierender Meinungen darüber, was genau als eine Strafnorm zu gelten hat, nicht eindeutig zu eruieren. Siehe zu diesen Schätzungen Larkin, Harvard Journal of Law \& Public Policy 37 (2014), 1065, 1091; Malcolm, The Pressing Need for Mens Rea Reform, Heritage Foundation, Legal Memorandum No. 160 vom 1.9.2015, 1.

2 Dazu anschaulich Stuntz, The Collapse of American Criminal Justice (2011).

3 Gerade rund um Duff entstanden zahlreiche Publikationen zu diesem Thema; siehe z.B. die Sammelwerke Duff u.a. (Hrsg.), The Boundaries of the Criminal Law (2010) und Duff u.a. (Hrsg.), Criminalization (2014).

4 So Koch/Holden, The Overcriminalization of America, POLITICO Online vom 7.1.2015, abrufbar unter: <www.politico.com/magazine/story/2015/01/overcrimi nalization-of-america-113991>. 
gelungsbereiche besteht, rücken aufgrund dieser Ausweitung der strafrechtlichen Normierungen und der Zuspitzung der sog. „Masseninhaftierung" jedenfalls die Grenzen des Strafrechts vermehrt ins Zentrum der Aufmerksamkeit. ${ }^{5}$

In diesem Zusammenhang führen gerade auch Fragen rund um die Anforderungen an das Schuldprinzip zu anhaltenden Diskussionen, da im gewachsenen Nebenstrafrecht der angelsächsischen Rechtsordnungen die sog. Strict Liability weite Verbreitung findet. Einer der Gründe für den „Strafrechtsexzess“ wird nämlich nicht nur in der rein quantitativen Ausweitung von Strafbestimmungen gesehen, sondern speziell auch in der beschränkten Geltung des Schuldprinzips im Rahmen dieser Doktrin der „strikten Verantwortlichkeit“. Die hohe Bedeutung dieser Strict Liability Offenses für die Overcriminalization-Debatte ist aus kontinentaleuropäischer Perspektive auf den ersten Blick nur schwer zu fassen. Während Lockerungen des Schuldprinzips auch hierzulande durchaus Anlass zu Kontroversen geben, handelt es sich bei der Strict-Liability-Doktrin um Einschnitte, wie sie deutschsprachigen Rechtsordnungen in diesem Ausmaß eindeutig fremd sind. Entsprechend war es im Rahmen der vieldiskutierten US-amerikanischen Criminal Justice Reform tatsächlich diese Doktrin, welche Anlass großer Differenzen war und schlussendlich sogar zu einem politischen Stillstand führte. ${ }^{6}$ Diese sog. Mens Rea Debate, bei der kaum die strafrechtstheoretische Verfechtung des Schuldprinzips, sondern vielmehr pragmatische Überlegungen im Zentrum standen, offenbart dabei deutlich, dass die konkrete Verankerung des Schuldprinzips hohe politische Relevanz erlangen kann. Dieser Konnex zwischen Overcriminalization und Schuldprinzip verdient deshalb eine besondere Untersuchung, welche den Kern der vorliegenden Abhandlung bildet.

Im vorliegenden Beitrag soll eruiert werden, ob dem Schuldprinzip bzw. dessen Lockerungen beim genauer zu beleuchtenden „Strafrechtsexzess" in der Tat eine entscheidende Rolle zukommt. Dabei wird zunächst zu diskutieren sein, was den Kern der aktuellen kriminalpolitischen Debat-

5 Dieses Ausweitungsphänomen wird auch in der deutschsprachigen Literatur thematisiert, wobei auch hier unklar bleibt, inwiefern auch qualitativ von einer Ausweitung gesprochen werden kann, ist doch das gewachsene Nebenstrafrecht für die Verurteilungen zu weiten Teilen eher von bescheidener Relevanz; dazu z.B. Killias u.a., Grundriss der Kriminologie, 2. Aufl. (2011), Rn. 809 f.

6 Siehe z.B. George, Can Bipartisan Criminal Justice Reform Survive in the Trump Era?, New York Times Online vom 6.6.2017, abrufbar unter: $<$ www.newyorker.com/ news/news-desk/can-bipartisan-criminal-justice-reformsurvive-in-the-trump-era>. 
te überhaupt ausmacht und inwieweit es der Schlagabtausch zur „Überkriminalisierung" tatsächlich vermag, die Problematik ausreichend zu erfassen. Nach einführenden Erläuterungen zur OvercriminalizationThematik (B.) und einem Blick auf die Criminal Justice Reform und die Mens Rea Debate (C.) soll entsprechend ausgeführt werden, inwiefern der behauptete Zusammenhang zwischen Overcriminalization und der Doktrin der Strict Liability seine Berechtigung hat (D). Diese politischen Dimensionen des Schuldprinzips offenbaren dabei einen Bedarf an weiterer Differenzierung im Sinne einer Diskussion darüber, ob es sich tatsächlich um ein Problem der Kriminalisierung selbst oder - gerade entgegen dem Begriff - eigentlich nicht vielmehr um ein Problem übermäßiger bzw. illegitimer Bestrafung handelt (E.). Münden soll der Beitrag in einem Resümee über die Relevanz des Schuldprinzips für die Grenzen und die Funktionsweise des Strafrechts und die Implikationen der aufgedeckten Zusammenhänge für die Kriminalpolitik in den USA und anderswo (F.)

\section{B. Overcriminalization - \\ Politisches Schlagwort oder reales Phänomen? \\ I. Das richtige „Maß an Strafrecht“ und die Schwierigkeiten einer Quantifizierung}

Die bereits einleitend verwendete Terminologie Overcriminalization bildet in den USA ein beliebtes Schlagwort, wobei der Neologismus im Allgemeinen die „Überbeanspruchung“ und den „Missbrauch“ des Strafrechts bezeichnen soll. ${ }^{7}$ Die zahlreichen Abhandlungen über das richtige „Maß an Strafrecht" indizieren jedoch bereits, dass die angemessenen Grenzen des Strafrechts kaum einfach auszumachen sind und eine normative Theorie der Strafrechtsgrenzen deshalb ein komplexes Unterfangen ist. ${ }^{8} \mathrm{Ob}$ es „zu viel Strafrecht“ gibt und was „zu viel Strafrecht“ bedeutet, ist demzufolge keineswegs einfach festzustellen und kann sich auf verschiedene

7 Larkin, The Extent of America's Overcriminalization Problem, Heritage Foundation, Legal Memorandum No. 121 vom 9.5.2014, 1.

8 Siehe z.B. die Beiträge von Richman, in: Klarman u.a. (Hrsg.), The Political Heart of Criminal Procedure (2012), 64 ff.; Duff u.a. (Hrsg.), Criminalization (Fn. 3); Ashworth, Ohio State Journal of Criminal Law 5 (2008), 407, 407 ff.; Kadish, American Criminal Law Quarterly 7 (1968), 17; Packer, Limits of the Criminal Sanction (1968). 
Weisen manifestieren. ${ }^{9}$ Die politische Essenz der OvercriminalizationKritik lässt sich jedoch gemäß Brown wie folgt zusammenfassen: Der Begriff erfasse die ,normative Beanstandung, dass der Staat zu viele Straftaten kreiere und Dinge kriminalisiere, die eigentlich keine Straftaten darstellen sollten“. ${ }^{10}$ Neben dieser allgemeinen Kritik an der Schaffung neuer Strafnormen weist die Debatte jedoch vielseitige Facetten auf: So bildet die Zunahme an Strafnormen gerade auf Bundesebene den Gegenstand der sog. Federalization-Kontroverse, wobei diese Tendenz mitunter auch als eine der Hauptursachen der quantitativen Ausweitung des Strafrechts in seiner Gesamtheit bezeichnet wird. ${ }^{11}$ Aber auch die seit den Neunzigerjahren vermehrte Tendenz, im Rahmen verschiedener "Tough on crime“Kampagnen neue Strafbestimmungen zu schaffen, oder die weitgehenden Ermächtigungen der Verfolgungsbehörden, im Rahmen von „Fill in the blanks"-Gesetzen Straftaten selbst genauer zu umschreiben, werden thematisiert. Ferner seien auch die immer höheren Strafandrohungen relevanter Teil des Phänomens und nicht zuletzt eben die noch genauer zu diskutierenden, unzureichenden Anforderungen an die innere Tatseite. ${ }^{12}$

Neben der Vielschichtigkeit der Frage, welche Sachverhalte strafrechtlich überhaupt zu erfassen sind, ist ein weiterer Grund für die Komplexität dieses Untersuchungsgegenstandes die Unklarheit darüber, was überhaupt Kriminalisierung bedeutet bzw. wie eine Überkriminalisierung überhaupt gemessen werden kann. Gemäß Dubber bedeute „etwas zu kriminalisieren" schlichtweg, etwas in den Bereich des Strafrechtssystems zu inkludieren. ${ }^{13}$ In der Regel wird „Kriminalisierung“ entsprechend einfach als legislatorischer Prozess verstanden und konsequenterweise Overcriminali-

$9 \mathrm{Zu}$ verschiedenen Varianten der „Überkriminalisierung“ siehe z.B. Molina, New Criminal Law Review 14 (2011), 123, 125.

10 Brown, Journal of Law, Economics and Policy 7 (2011), 658, 658.

11 Als „Federalization“ wird dabei die kontinuierliche Ausweitung des Bundesstrafrechts thematisiert, wobei diese Expansion zu einem unübersichtlichen $\mathrm{Ma} \beta$ an materiellen Bundesstrafnormen geführt hat. Als „Föderalisierung“ wird das deshalb kritisiert, da der Bund mittlerweile eine faktische, in diesem Ausmaß bei der legislativen Aufgabenteilung ursprünglich nicht intendierte Strafgesetzgebungskompetenz erlangt hat, was in einer weitgehenden Überlagerung des Strafrechts der Bundesstaaten und des Bundes resultierte. Siehe zu dieser Federalization Debate ausführlich und m.w.N. Neumann, Das US-amerikanische Strafrechtssystem als Modell für die vertikale Kompetenzverteilung im Strafrechtssystem der EU? (2014), insb. 41 ff., 78 ff., $126 \mathrm{ff}$.

12 Larkin (Fn. 7), 1.

13 Dubber, in: Duff u.a. (Hrsg.), Boundaries (Fn. 3), 191, 191. 
zation als ein „Problem der legislatorischen Überaktivität“.${ }^{14} \mathrm{Gemäß} \mathrm{die-}$ ser positivistischen Grundhaltung ist eine Straftat also einfach, was Gegenstand einer Strafbestimmung ist und deshalb von den Behörden strafrechtlich verfolgt werden kann, das heißt eine sanktionsbewehrte Normierung von Verhaltensweisen. ${ }^{15}$ Andere Autoren wie Lacey unterscheiden hingegen zwischen formal criminalization (,law in books") und substantive criminalization (, law in action") ${ }^{16}$ wobei letztere nicht immer auf erstere folgen müsse. ${ }^{17}$ Auch wenn diese Unterscheidung ihre Berechtigung hat und für die Beurteilung der Overcriminalization-Problematik durchaus zwischen der rein formalen Ausweitung von Strafbestimmungen und der effektiven Durchsetzung derselben differenziert werden sollte, muss darüber hinaus ebenso berücksichtigt werden, dass bereits eine rein formale Kriminalisierung nicht irrelevant ist. So kann eine solche auch ohne effektive Strafverfolgung Konsequenzen für die soziale Ordnung und das Verhalten der Bürger haben. ${ }^{18}$ Kriminalisierung zu quantifizieren ist folglich keineswegs ein leichtes Unterfangen, ist doch die reine Angabe der Zahl an Strafbestimmungen zwar ein wesentlicher Indikator, liefert diese aber dennoch kaum ein abschließendes Bild. ${ }^{19}$ Es sollte jedoch bereits jetzt deutlich werden, dass die Diskussionen rund um die Mass Incarceration und diejenigen rund um die (formale) Kriminalisierung zu differenzieren sind, wobei sowohl Zusammenhänge als auch Differenzen noch genauer unter die Lupe genommen werden müssen.

\section{US-amerikanische Eigenheiten und die Ausweitung des Strafrechts}

Bereits ein summarischer Blick auf die sehr zahlreichen Seiten der verschiedenen Strafgesetze der US-Bundestaaten lässt erkennen, dass in der Tat vieles kriminalisiert wird, was in Kontinentaleuropa kaum je den Weg in ein Strafgesetz finden würde. Gemäß Luna, der eindrücklich dutzende absurde Kriminalisierungen aufführt, würden gewisse dieser Bestimmun-

14 Duff u.a., in: Duff u.a. (Hrsg.), Criminalization (Fn. 3), 6.

15 Vgl. z.B. Pettit, in: Duff u.a. (Hrsg.), Criminalization (Fn. 3), 132, 133.

16 Lacey, Modern Law Review 72 (2009), 936, 943.

17 Chalmers/Leverick, in: Duff u.a. (Hrsg.), Criminalization (Fn. 3), 54, 55.

18 Vgl. Chalmers/Leverick (Fn. 17), 56. Auch empirisch ließ sich eine Wirkung der Einführung neuer Strafbestimmungen immer wieder nachweisen; siehe dazu Killias u.a. (Fn. 5), Rn. 1025 ff. m.w.N.

19 Bereits das eigentliche „Zählen“ von Strafbestimmungen ist Gegenstand von Kontroversen; dazu Duff u.a., in: Duff u.a. (Hrsg.), Criminalization (Fn. 3), 7. 
gen nicht einmal den „Lachtest“ bestehen, da ihnen jede legitime Grundlage fehlen würde - und man eben oftmals sogar lachen müsse. ${ }^{20}$ Die Anzahl der Straftatbestände und die jeweiligen Regelungsgegenstände geben demnach in der Tat Anlass zu berechtigter Kritik. ${ }^{21}$

Es ist dabei das Bewusstsein wichtig, dass sich die US-amerikanische Debatte kaum mit den auch in verschiedenen kontinentaleuropäischen Rechtsordnungen geführten Diskussionen über die Überbeanspruchung des Instruments der Pönalisierung vergleichen lässt. Gemäß Molina seien uns die meisten Fragen der amerikanischen Overcriminalization-Krise schlichtweg unbekannt, da wir die zugrundeliegenden Probleme bereits anderweitig gelöst hätten. ${ }^{22}$ Tatsächlich ist eine der plausibleren Erklärungen für den weiteren Anwendungsbereich des Strafrechts in den USA, dass den Gefahren, welche sich gerade aus dem rasanten technologischen Fortschritt ergaben, in Europa vermehrt mit anderen Kontrollstrategien als derjenigen des strafrechtlichen Schuldvorwurfs begegnet wurde. Anderweitige, im Vorfeld wirkende Regulierungen sollen so bereits die Möglichkeit individuellen Fehlverhaltens eliminieren. ${ }^{23}$ Die US-amerikanische Politik ist hingegen generell zurückhaltender mit staatlichen Eingriffen und kollektiven Maßnahmen. Das Strafrecht wird so auch zur Konsequenz eines „schwachen Staates“, welcher einem individualistischen Ansatz folgend erst dann eingreifen soll, wenn bereits negative Auswirkungen ein Eingreifen erforderlich machen. ${ }^{24}$ Dieses auch als ,govern through crime“ kritisierte Phänomen ${ }^{25}$ ist sicherlich kein rein US-amerikanisches, findet dort jedoch vermehrt Rückhalt. Molina führt als eine der möglichen Erklärungen sodann auch gleich den bedeutend höheren Stellenwert an, den das Schuldprinzip in Kontinentaleuropa genieße. ${ }^{26}$ Überhaupt sei die ausgeprägte Lehre des Allgemeinen Teils des Strafrechts und die Prinzipienorientiertheit der Strafrechtsordnungen dafür ausschlaggebend, dass bereits an den Universitäten jeder und jedem Jura-Studierenden klar werde, dass

20 Luna, American University Law Review 54 (2005), 703 f. Als Beispiel führt Luna z.B. eine Bestimmung aus dem Bundesstaat Alabama an, nach der zu bestrafen sei, wer einen Bären für Ringkämpfe trainiere.

21 Zur Anzahl der Straftatbestände siehe bereits Fn. 1 mit den entsprechenden Nachweisen.

22 Molina (Fn. 9), 124.

23 Vgl. Killias u.a. (Fn. 5), Rn. 812.

24 Vgl. Richman (Fn. 8), 69 ff.

25 Siehe z.B. Lacey (Fn. 16), 939

26 Molina (Fn. 9), 127. 
Strict Liability Offenses aufgrund ihres Widerspruchs zum Schuldprinzip nicht akzeptabel seien. ${ }^{27}$

Unabhängig der genauen, in der Folge noch zu erörternden Ursachen für das als zu hoch kritisierte „Maß an Strafrecht“ lässt sich nicht bestreiten, dass der unter dem Titel Mass Incarceration behandelte exponentielle Anstieg der Gefängnispopulation seit den Achtzigerjahren die Kriminalpolitik vor Herausforderungen stellt. Das mit der OvercriminalizationDebatte eng verflochtene Phänomen findet hier eine eindrückliche Zahlengrundlage, welche die Relevanz und das Drängen auf verschiedene Strafrechtsreformen sichtlich nachvollziehbar macht. Waren 1980 nämlich noch rund 300.000 Personen in US-amerikanischen Gefängnissen inhaftiert, waren es 1990 bereits 800.000, 2000 schon 1.4 Millionen und nochmal 10 Jahre später wurde mit 1.6 Millionen der vorläufige Höchstwert erreicht (siehe Grafik unten). ${ }^{28}$ Diese deutliche Zunahme hat mittlerweile zu einem breiten Konsens geführt, dass Reformen angezeigt sind. ${ }^{29}$ Der leichte Rückgang im aktuellen Jahrzehnt ist sodann auch bereits auf Reformbestrebungen einiger Bundesstaaten zurückzuführen, ${ }^{30}$ wobei die sogleich zu diskutierende Criminal Justice Reform nach wie vor politisches Dauerthema ist. Auch im internationalen Vergleich westlicher Staaten sind diese Zahlen beachtlich. Für das Jahr 2015 konnte für die USA eine Inhaftierungsrate von 458 Insassen pro 100.000 Einwohner errechnet werden. ${ }^{31}$ Zum Vergleich: In Deutschland betrug die Rate im gleichen Jahr 77,4, in der Schweiz 82,7 und in Österreich 103,9. ${ }^{32}$ Deutlich wird sowohl bei einem Blick auf diese Zahlen als auch auf die Zunahme von Strafbestimmungen, dass die Debatte um eine Overcriminalization ihre Berechtigung hat. Auch wenn zwischen der eigentlichen Ausweitung der Kriminalisierung auf der einen und der tatsächlichen Anwendung des Strafrechts (und dessen Konsequenzen) auf der anderen Seite differenziert werden muss, ist zu erkennen, dass eine gewisse „Überbeanspruchung“ und ein „zu hohes $\mathrm{Ma} ß$ an Strafrecht" kaum geleugnet werden können.

27 Molina (Fn. 9), 131.

28 U.S. Bureau of Justice Statistics, National Prisoner Statistics, 1978-2015, abrufbar unter: $<$ www.bjs.gov/index.cfm?ty=pbdetail\&iid $=5869>$.

29 Siehe z.B. Liptak, Right and Left Join Forces on Criminal Justice, New York Times Online vom 23.11.2009, abrufbar unter: <https://nyti.ms/2k764FY>.

30 Siehe zu diesen Maßnahmen z.B. Lampard, in: The State Factor, ALEC, November 2016 .

31 U.S. Bureau of Justice Statistics (Fn. 28).

32 Siehe Council of Europe Annual Penal Statistics: SPACE, Annual Report 2015, abrufbar unter: <www.coe.int/en/web/prison/space $>$. 


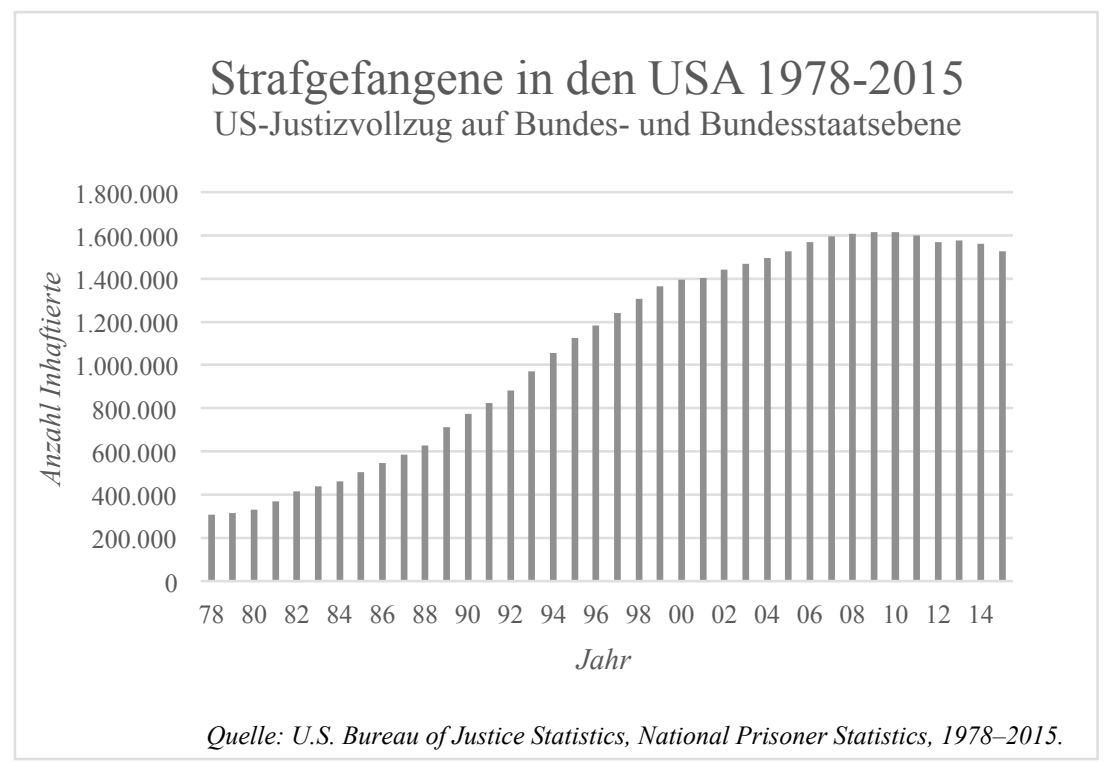

C. Relevanz der Mens-Rea-Debatte für die Criminal Justice Reform

I. Strafrechtsreform und neue kriminalpolitische Überparteilichkeit

Als Resultat der anhaltenden Overcriminalization-Debatte, welche nicht nur die Strafrechtswissenschaft, sondern vor allem auch die Politik seit Jahren beschäftigt, standen in neuester Zeit konkrete Reformprojekte auf der politischen Agenda in Washington D.C. Während unter dem Titel Criminal Justice Reform in der Vergangenheit mehrere Reformunterfangen diskutiert wurden, steht hier die neuere Bestrebung im Zentrum, den Problemen der Strafrechtsausweitung, vor allem aber auch der Masseninhaftierung, Herr zu werden. Vordergründig wird dabei die Reduktion der Gefängnispopulation angestrebt, unter anderem mittels einer Sentencing Reform, das heißt einer Reform der Strafandrohungen wie zum Beispiel der sog. Mandatory Minimum Sentences im Bereich weniger schwerwiegender Drogendelikte. Während verschiedene Bundesstaaten bereits solche Reformen umgesetzt haben, ${ }^{33}$ ist die Debatte auf Bundesebene noch

33 Vgl. z.B. die Übersicht der Maßnahmen im Jahr 2016: Lampard (Fn. 30). 
nicht abgeschlossen und trotz weitreichender Kompetenzen der Bundesstaaten keineswegs von untergeordneter Bedeutung.

Während Anläufe liberaler Strömungen, kriminalpolitisch neue Wege einzuschlagen, lange Zeit keine politischen Mehrheiten fanden, etablierte sich in den letzten Jahren ein neuer politischer Konsens, welcher das Ende eines langen politischen Stillstands zu versprechen schien. Diese neue Criminal Justice Reform wurde entsprechend von Vertretern der beiden US-politischen Lager getragen und gilt daher grundsätzlich als ,überparteiliches" Unterfangen. ${ }^{34}$ Auch wenn die konkreten Interessenlagen und Bedingungen bezüglich der Reform im Detail variieren, ist diese überparteiliche Einigkeit, dass in Bezug auf das Strafrecht und den Strafvollzug großer Reformbedarf besteht, beachtlich, wenn man die verhärteten Fronten seit der sog. Crime Wave bedenkt. ${ }^{35}$ Dass konservative Kräfte im Vergleich zu früheren Dekaden nicht mehr nur härteres Durchgreifen fordern, kann dabei auf verschiedene Ursachen zurückgeführt werden: Neben finanziellem Druck und einer tatsächlichen Entschärfung des Kriminalitätsaufkommens im neuen Jahrtausend ließ sich durchaus auch ein tatsächliches ideologisches Umschwenken verschiedener Akteure beobachten. ${ }^{36}$

Der neu erlangte Konsens, der Masseninhaftierung müsse nun Einhalt geboten werden, wurde jedoch schnell von Uneinigkeiten rund um Einzelheiten überschattet. Einmal abgesehen davon, dass im Moment unklar scheint, inwiefern sich die neue Trump-Regierung auf das Unterfangen auswirkt, ${ }^{37}$ scheiterte das Vorhaben während der letzten Amtsperiode bereits an einem anhaltenden Dissens über die Notwendigkeit einer gleichzeitig zu verabschiedenden sog. Mens Rea Reform. In den USA wird die

34 Siehe dazu z.B. The Marshall Project, Nine Lessons About Criminal Justice Reform, 19.9.2017, abrufbar unter: <www.themarshallproject.org/2017/07/19/ninelessons-about-criminal-justice-reform>.

35 Ein zu beobachtender Anstieg der Kriminalität führte seit den Siebzigerjahren zu einem punitiven Klima, und es herrschte weitgehende Einigkeit, dass dem Problem mit entsprechender Härte begegnet werden müsse, was u.a. auch die Kluft zwischen den gegenläufigen Entwicklungen der Gefangenenraten in Europa und den USA zu erklären vermag; siehe dazu Kleinfeld, Stanford Law Review 68 (2016), 933, 937 m.w.N.

$36 \mathrm{Zu}$ diesem Umschwung in konservativen Staaten und in der republikanischen Partei ausführlich Dagan/Teles, Prison Break. Why Conservatives Turned Against Mass Incarceration (2016).

37 Trump hat zur Strafrechtsreform noch wenig Konkretes verlautbaren lassen. Dennoch gelten sowohl er als auch sein Generalstaatsanwalt allgemein als kriminalpolitische „Hardliner“ und als Befürworter von „Tough on crime“Maßnahmen. Siehe dazu z.B. George (Fn. 6). 
Overcriminalization-Thematik, wie bereits einleitend erwähnt, offen mit der Diskussion rund um die Strict Liability und damit mit dem Schuldprinzip verknüpft. ${ }^{38}$ Trotz der Breite an diskutierten Maßnahmen im Rahmen der Reform war es am Ende in der Tat dieser Punkt, welcher überraschende Relevanz erlangte und der neu erzielten politischen Einigkeit einen Dämpfer verpasste.

\section{Schuldprinzip und das vorläufige Scheitern der Strafrechtsreform}

Dass der Kampf gegen Overcriminalization und Mass Incarceration ausgerechnet an der Frage nach der Legitimität der strikten Verantwortlichkeit bzw. der Einhaltung des Schuldprinzips vorläufig scheiterte, verblüfft auf den ersten Blick. Der Umstand, dass die Reform heute wieder auf Eis liegt und nicht mehr wie geplant während der Obama-Präsidentschaft verabschiedet werden konnte, ist dabei jedoch tatsächlich zu einem wesentlichen Teil auf die Forderungen in Bezug auf die Mens Rea Reform zurückzuführen. ${ }^{39}$ Primär konservative Kräfte vertreten den Standpunkt, dass die konsequente Einhaltung des Schuldprinzips eine der wirksamsten Maßnahmen gegen die Überbeanspruchung des Strafrechts wäre. So sollten die von republikanischen Abgeordneten vorgeschlagenen Mens Rea Reform Act 2015 und Criminal Code Improvement Act 2015 zumindest für das Bundesstrafrecht im Bereich des Gesundheits-, Arbeits- und Umweltschutzes die Geltung des Schuldprinzips garantieren und einheitliche Mens-Rea-Erfordernisse festlegen. ${ }^{40}$ Nur eine zeitgleiche Verabschiedung einer solchen Reform, welche der Strict Liability im Bereich eben dieser sog. Public Welfare Offenses Einhalt gebieten solle, würde ihre Unterstützung für die eigentliche Reform der Strafrechtspflege sichern. Die Reform sei notwendig, da ein angemessenes Schulderfordernis als Voraussetzung von Strafbarkeit eine der größten Garantien gegen die Overcriminalization sei. Während sich die Reform der Sanktionen und Strafkataloge der Frage annehmen würde, wie lange Verurteilte eine Strafe verbüßen müssen,

38 Siehe dazu z.B. Thompson, Mens Rea Reform: A Brief Overview, Congressional Research Service, 14.4.2016, $1 \mathrm{ff}$.

39 Dazu z.B. Sarch, How to solve the biggest issue holding up criminal justice reform, POLITICO Online vom 6.15.2016, abrufbar unter: $<$ www.politico.com/agen da/story/2016/05/criminal-justice-reform-mens-reamiddle-ground-000120>.

40 So z.B. die Stellungnahme von Malcolm (Fn. 1), 1 ff., als Vertreter eines konservativen Think Tanks. 
würde die Mens Rea Reform bereits bei einer der Ursachen des Problems ansetzen: dass moralisch Unschuldige gar nicht erst durch das Strafrechtssystem verfolgt würden. ${ }^{41}$ Kritiker dieser Position wiederum sehen die Essenz dieser Mens Rea Reform einzig darin, dass Republikaner es damit erschweren wollten, Wirtschaftskriminalität zu verfolgen. ${ }^{42}$ Demokraten wehren sich deswegen weitgehend gegen die Forderungen, die Ansprüche an den Nachweis der inneren Tatseite im Bereich dieser Public Welfare Offenses anzuheben, da dies die Verfolgung von Unternehmen und Unternehmensverantwortlichen erschweren würde. ${ }^{43}$

Kritiker der Overcriminalization, darunter allen voran die Heritage Foundation - ein einflussreiches konservatives Think Tank und Forschungsinstitut -, weisen auf Daten hin, die belegten, dass vor allem in Wahljahren deutlich mehr neue Straftatbestände geschaffen würden. Dies sei vor allem deshalb problematisch, da die Parlamente gleichzeitig die Rolle der inneren Tatseite und des Schuldprinzips zunehmend schwächen würde, was die „Macht des Strafrechts als Instrument der Moral Educati$o n^{\text {" }}$ reduziere. ${ }^{44}$ So würden zwar die Mehrheit der neuen Strafgesetze jeweils ein Mens-Rea-Erfordernis wie knowingly oder willfully enthalten, trotzdem sei bei einem beachtlichen Teil auf ein solches Erfordernis verzichtet und seien damit neue Strict Liability Offenses geschaffen worden. ${ }^{45}$ Die konservativen Kritiker fokussieren sich dabei allerdings nur auf die Public Welfare Offenses und heben hervor, dass das Schuldprinzip gerade in diesem Bereich Personen schützen solle, welche aus Versehen oder unbewusst das Recht brechen. ${ }^{46}$ Auf die Lockerungen des Schuldprinzips im eigentlichen Kernstrafrecht gehen sie dabei nicht ein und lassen damit wie noch genauer zu diskutieren sein wird - einen wesentlichen Punkt der Debatte um die Doktrin der Strict Liability unberücksichtigt.

$41 \mathrm{Zu}$ diesen Argumenten Malcolm (Fn. 1), 1.

42 Siehe z.B. Heitzeg, Criminal InJustice: The Corporate „Person“, Mens Rea and the Right Wing „Reform“ Ruse, Critical Mass Progress Online vom 10.2.2016, abrufbar unter: <http://criticalmassprogress.com/2016/02/10/criminal-injusticethe-corporate-person-mens-rea-and-the-right-wing-reform-ruse $>$.

43 Dazu z.B. Thompson (Fn. 38), 1; Sarch (Fn. 39).

44 Baker, Revisiting the Explosive Growth of Federal Crimes, Heritage Foundation, Legal Memorandum No. 26 vom 16.6.2008.

45 Baker (Fn. 44), $6 \mathrm{f}$

46 Baker (Fn. 44), 6. 


\section{Mens Rea Reform zwischen Theorie und politischem Kalkül}

Die Skepsis der Demokraten diesen Forderungen gegenüber wird wohl auch dadurch bestärkt, dass die größten Treiber hinter der Mens Rea Reform nicht nur die Heritage Foundation, sondern auch die Gebrüder Charles G. und David H. Koch sind, deren Familie die Koch Industries kontrolliert, das zweitgrößte US-Unternehmen in Privatbesitz. ${ }^{47}$ Deren konservative und libertäre Koch Family Foundation hat sich im Rahmen der Strafrechtsreform besonders hervorgetan, wobei die Einflussnahme der Milliardäre auch Misstrauen weckte, wenn sie auch zweifellos dem Unterfangen mehr politisches Gewicht gaben. ${ }^{48}$ Der Einsatz der Kochs für die Sentencing Reform, welche dann aber durch die republikanische Forderung nach der Mens Rea Reform torpediert wurde, wird entsprechend kritisch gesehen und die Reform deshalb auch als "Corporation-Protecting Mens Rea Reform" bezeichnet. ${ }^{49}$ Dieses Misstrauen erhärtet sich noch, wenn man die weitere Liste der Unterstützer betrachtet. Ob die Chamber of Commerce oder die National Federation of Independent Business: Die Bekämpfung der Strict Liability im Nebenstrafrecht und dabei vor allem auch im Wirtschaftsstrafrecht erhält wenig überraschend viel Unterstützung aus (konservativen) Unternehmerkreisen, welche die Möglichkeiten der Strafverfolgung von ihresgleichen eindämmen wollen. ${ }^{50}$ Gerade im Bereich der Strafnormen zum Schutz der öffentlichen Gesundheit und Sicherheit und des Umweltschutzes sei der vorgeschlagene Mens Rea Reform Act of 2015 problematisch, weshalb sich mehr als 200 amerikanische NGOs dagegen ausgesprochen haben, unter anderem auch mit dem Verweis darauf, dass damit das Problem der Overcriminalization keineswegs richtig adressiert sei. ${ }^{51}$

47 Murphy, America's Largest Private Companies 2017, Forbes Online vom 9.8.2017, abrufbar unter: <www.forbes.com/sites/andreamurphy/2017/08/09/am ericas-largest-private-companies-2/\#757f7ba9247c > .

48 Ball, Do the Koch Brothers Really Care About Criminal-Justice Reform?, The Atlantic Online vom 3.3.2015, abrufbar unter: $<$ www.theatlantic.com/politics/archive /2015/03/do-the-koch-brothers-really-care-about-criminal-justicereform/386615>.

49 Siehe dazu die Schilderungen des demokratischen Senators Sheldon Whitehouse in seinem Buch: Captured. The Corporate Infiltration of American Democracy (2017), insb. Kapitel 4.

50 Edsall, Legislation by Stealth, Republican Style, New York Times Online vom 20.4.2016, abrufbar unter: <https://nyti.ms/1SuyFEg>.

51 Siehe dazu den Brief der sog. Leadership Conference vom 19.1.2016, abrufbar unter: $<$ http://civilrights.org/the-leadership-conference-on-civil-and-human-right2016-legislative-priorities-senate>. 
Dennoch sollen diese republikanische Forcierung der Mens Rea Reform und das entstandene Misstrauen gemäß Kommentatoren nicht darüber hinwegtäuschen, dass eine solche Reform eine notwendige Verbesserung darstellen würde. ${ }^{52}$ So hätten beispielsweise gemäß Sarch beide Seiten legitime Argumente: Eine Mens Rea Reform würde in der Tat rechtliche Klarheit schaffen und könnte ungerechte Strafverfolgungen verhindern. Andererseits gingen die Republikaner in ihren Vorschlägen zu weit und stellten zu hohe Anforderungen an den Schuldnachweis, so dass viele durchaus Verantwortliche nicht mehr verfolgt werden könnten. Entsprechend solle ein Mittelweg gefunden werden und bereits eine leichte Form von Fahrlässigkeit ausreichen. ${ }^{53}$

Bereits hier zeigt sich deutlich die politische „Aufladung“ der Debatte um das Schuldprinzip. Dennoch ist eine sachliche Beurteilung der umrissenen Positionen - abseits von politischen Ränkespielchen - kaum möglich, solange nicht klar ist, um was es bei der Strict Liability genau geht, was sie beinhaltet und inwiefern sie tatsächlich mit der Overcriminalization-Problematik zusammenhängt. Bevor also die Forderung der Republikaner, dem Schuldprinzip bei den Public Welfare Offenses zum Durchbruch zu verhelfen, beurteilt werden kann - eine aus strafrechtswissenschaftlicher Sicht im Allgemeinen einleuchtende Forderung -, muss entsprechend in der Folge zunächst ein genauerer Blick auf die im Zentrum stehende Doktrin der strikten Verantwortlichkeit geworfen werden.

\section{Strict Liability Offenses und die Politik des Schuldprinzips}

\section{Verankerung des Schuldprinzips im US-amerikanischen Strafrecht}

Um ein besseres Verständnis für die bereits skizzierten Diskussionen rund um die Strafrechts- und Mens-Rea-Reform zu erhalten, ist es zunächst notwendig, das Fundament der US-amerikanischen Strafrechtsdoktrin und dabei vor allem auch die Bedeutung des Schuldprinzips genauer zu beleuchten. Das Schuldprinzip findet im Strafrecht der USA keine positivrechtliche Grundlage, gilt aber trotz der zum Teil gewichtigen Durchbrechungen im Rahmen der bereits mehrfach erwähnten Strict-Liability-

52 Siehe z.B. Yaffe, A Republican Crime Proposal That Democrats Should Back, New York Times Online vom 12.2.2016, abrufbar unter: $<$ https://nyti.ms/2jHC MQQ>.

$53 \operatorname{Sarch}$ (Fn. 39). 
Doktrin als eines der tragenden Rechtsprinzipien. ${ }^{54}$ Actus non facit reum nisi mens sit rea - die Handlung allein begründet nicht die Strafbarkeit, sie muss vielmehr begleitet sein von der Schuld - heißt sodann auch der wohl berühmteste Satz der angelsächsischen Zurechnungslehre und bildet damit das Pendant zum in Kontinentaleuropa gebräuchlicheren Lehrsatz nulla poena sine culpa ${ }^{55}$ Der auch als Mens Rea-Prinzip bezeichnete Grundsatz kann allerdings hinsichtlich Bedeutung und Gehalt kaum mit dem Verständnis des Schuldprinzips, wie es in deutschsprachigen Rechtsordnungen vorherrscht, verglichen werden. ${ }^{56} \mathrm{Im}$ Gegensatz zu Deutschland ${ }^{57}$ wurde das Schulderfordernis bzw. das Erfordernis einer inneren Tatseite bislang auch nicht als verfassungsrechtlich unabdingbares Element der Strafbarkeit anerkannt. ${ }^{58}$ Der US-amerikanische Supreme Court hat immer wieder festgehalten, dass Mens Rea zwar ein wichtiges Erfordernis sei, allerdings kein verfassungsrechtlich garantiertes, weshalb auch Ausnahmen möglich seien. ${ }^{59}$

Wenn man aus deutschsprachiger Perspektive über den Stellenwert des Schuldprinzips im angelsächsischen Recht spricht, ist es ferner wichtig, bei dieser Gegenüberstellung die Unterschiede der Straftatlehren zu berücksichtigen. Während sich im deutschsprachigen Raum die SchuldUnrecht-Dichotomie gerade durch die finale Handlungslehre ${ }^{60}$ durchgesetzt hat und eine konsequente Differenzierung zwischen Schuld im engeren Sinne und subjektivem Tatbestand vorgenommen wird, ist eine vergleichbare Entwicklung in den Ländern des Common Law nicht erkenn-

54 Dazu z.B. Vogel, GA 1998, 127 ff.; Simmler, Normstabilisierung und Schuldvorwurf. Eine Analyse der Funktion und Variabilität der strafrechtlichen Schuld am Beispiel der Strict Liability (2018), 50 ff.

55 Siehe zu diesem Grundsatz (und sogleich auch den Ausnahmen) auch Bähr, Strafbarkeit ohne Verschulden (Strict Liability) im Strafrecht der USA (1974), 23; Hörster, Die strict liability des englischen Strafrechts (2009), 5; Tully, Poena sine Culpa? - Strict-Liability-Sanktionen und Europäisches Gemeinschaftsrecht (2000), 23.

56 So auch Safferling, Vorsatz und Schuld (2008), $481 \mathrm{f}$.

57 BGHSt 2, 194, 200.

58 Weik, Objektive und subjektive Verbrechenselemente im US-amerikanischen Strafrecht (2004), 439 mit Verweis auf Powell v. Texas, 392 U.S. 514, 535 f. (1968)

59 Dazu Dubber/Hörnle, Criminal Law. A Comparative Approach (2014), 102; Singer, Boston College Law Review 30 (1989), 337, 403; ausführlich auch Singer/Husak, Buffallo Criminal Law Review 2 (1999) 861.

60 Welzel, Das Deutsche Strafrecht, 11. Aufl. (1969), 140 und passim. 
bar. ${ }^{61}$ In der Tradition der stärkeren prozeduralen Ausrichtung der Straftatlehre wird zwischen Actus Reus, Mens Rea und Defenses unterschieden. Mens Rea wird im Englischen mit guilty mind, das heißt schuldiger Geist, übersetzt, sogleich aber regelmäßig präzisiert, dass sich die rechtliche Verwendung des Begriffs auf einen Geisteszustand hinsichtlich eines konkreten Delikts beziehe. ${ }^{62}$ Die Frage, was genau Mens Rea bedeutet, ist allerdings umstritten. In der Regel werden beim Mens Rea die verschiedenen Formen des Vorsatzes und der Fahrlässigkeit geprüft, während Schuldminderungs- und Schuldausschlussgründe erst bei den Defenses zum Tragen kommen. ${ }^{63}$ Da kein allgemeines - mit einem Allgemeinen Teil deutschsprachiger Strafgesetzbücher vergleichbares - Konzept in Bezug auf das Schulderfordernis existiert muss im Rahmen der Mens-ReaIdentifikation für jedes Delikt einzeln bestimmt werden, welche Ansprüche an die innere Tatseite zu stellen sind, wobei der amerikanische Bundesgerichtshof dafür eine Reihe von Vermutungen und Hilfestellungen entwickelt hat, um im Einzelfall festzustellen, ob überhaupt und welche Mens Rea-Form verlangt ist. ${ }^{64}$ Auf diesen Umstand sind auch die große Variationsbreite an Anforderungen und die zahlreichen Unklarheiten in diesem Bereich zurückzuführen, welche sich auch in der Mens Rea Debate niederschlagen.

\section{Doktrin der strikten Verantwortlichkeit}

Die historischen Wurzeln der strikten Verantwortlichkeit sind nicht ganz klar, werden jedoch in der Regel auf die Mitte des 19. Jahrhunderts zurückdatiert, wobei deren Verbreitung primär mit der Industrialisierung und dem damit einhergehenden erhöhten Regulierungsbedürfnis in Zusammenhang gebracht wird. ${ }^{65}$ Die Industrialisierung habe zu einer deutlichen

61 Welzels finale Handlungslehre findet in der angelsächsischen Literatur nur Erwähnung als Lehre, die „ein deutscher Philosoph“ vorgelegt habe; so Vogel (Fn. 54), $137 \mathrm{ff}$.

62 Norrie, Crime, Reason and History, 2. Aufl. (2001), 35.

$63 \mathrm{Zu}$ den verschiedenen Formen der Mens Rea siehe z.B. Dubber, An Introduction to the Model Penal Code, 2. Aufl. (2015), 35 ff.; Allen, Textbook on Criminal Law, 12. Aufl. (2013), $58 \mathrm{ff}$.

64 Dazu z.B. Thompson (Fn. 38), Summary.

65 Sayre, Columbia Law Review 33 (1933), 55, 56; Greenberg/Brotman, American Criminal Law Review 51 (2014), 79, 79; Hörster (Fn. 55), 25; Safferling (Fn. 56), 374 f.; Singer (Fn. 59), 345; Weik (Fn. 58), 241. 
Ausweitung der Strafgesetzgebung geführt und das Bedürfnis erhöht, das Strafrecht nicht mehr nur zur Wahrung von Individual-, sondern zunehmend auch von Kollektivinteressen einzusetzen. Die Allgemeinheit sollte gerade durch diese neu geschaffenen Public Welfare Offenses vor negativen Auswirkungen geschützt werden. ${ }^{66}$ Bei diesen Delikten ist in der Folge immer häufiger auf den vollständigen Nachweis einer inneren Tatseite verzichtet worden, was primär mit pragmatischen Argumenten der Prävention bzw. der erleichterten Strafverfolgung legitimiert wurde ${ }^{67}$ Allerdings ist hierbei zu beachten, dass sich gerade in den USA die strikte Verantwortlichkeit keineswegs nur im Bereich dieses Nebenstrafrechts ausgebreitet hat, sondern auch bei Delikten, bei welchen die Moral bzw. die Kriminalisierung von Verhaltensweisen, die als moralisch besonders anstößig galten, im Zentrum standen. ${ }^{68}$ Die Strict Liability Offenses sind nach wie vor weit verbreitet, und der Hinweis, es handle sich dabei nur um „letzte Inseln“ oder ,archaische Relikte“ und nicht um „typisch amerikanisches Strafrecht", ist zweifellos unpassend. ${ }^{69}$ Daran ändert auch der Umstand nichts, dass die Doktrin einer permanenten wissenschaftlichen Kritik ausgesetzt ist. $^{70}$

Obwohl in den USA also allgemein durchaus gilt, dass nur Schuld Strafe legitimieren kann, ist dieser Grundsatz in der Praxis zahlreichen Durchbrechungen ausgesetzt, welche weit über das hinausgehen, was wir im deutschsprachigen Raum als Lockerungen des Schuldprinzips zu diskutieren gewohnt sind. ${ }^{71}$ Die Definitionsversuche dieser sog. strikten Verantwortlichkeit divergieren allerdings beträchtlich, und der Begriff wird in der Literatur nicht einheitlich verwendet. ${ }^{72}$ Einerseits wird Strict Liability oftmals einfach als „Verantwortlichkeit ohne Schuld“ bezeichnet oder als Strafbarkeit, bei welcher der Nachweis von Vorsatz oder Fahrlässigkeit

66 Bähr (Fn. 55), 80; Greenberg/Brotman (Fn. 65), 80; Sayre (Fn. 65), 67.

67 Hörster (Fn. 55), 26 und 30; zu diesen Argumenten siehe auch Simmler (Fn. 54), $62 \mathrm{f}$. und $86 \mathrm{ff}$.

68 Singer (Fn. 55), 339; Weik (Fn. 58), 241 f.

69 So aber Quist, Die Public Welfare Offences im Amerikanischen Strafrecht (1971), 3, in Bezug auf die Verbreitung im Kernstrafrecht.

70 Siehe z.B. Larkin (Fn. 1), 1079; Simester u.a., Simester and Sullivan's Criminal Law, 5. Aufl. (2013), 192 f.; Michaels, Harvard Law Review 223 (1999), 828, 831, m.w.N.; Singer (Fn. 59), 403 ff.; Tadros, Criminal Responsibility (2005), 73 f.; Wasserstrom, Stanford Law Review 12 (1960), 731, $731 \mathrm{f}$.

71 Ausführlich zu möglichen Erklärungen für diese Differenzen zwischen den Rechtsordnungen Simmler (Fn. 54), 79 ff.

72 Vgl. auch Green, in: Simester (Hrsg.), Appraising Strict Liability (2005), 1, 2; zu diesen Definitionsversuchen auch Simmler (Fn. 54), 54 ff. 
nicht von Relevanz sei. ${ }^{73}$ Diese Definition als Verantwortlichkeit ohne jedes Verschulden wird jedoch weitgehend als praxisfern und zu eng abgelehnt. ${ }^{74}$ Das gebräuchlichere Begriffsverständnis ist präziser und lautet dahingehend, dass es sich bei Strict Liability Offenses um Delikte handle, bei welchen für mindestens ein objektives Tatbestandsmerkmal kein korrespondierender subjektiver Tatbestand nachgewiesen werden müsse. ${ }^{75}$ Auch wenn in der Tat den meisten Strict-Liability-Delikten solche Lockerungen hinsichtlich des subjektiven Tatbestands inhärent sind, trifft auch diese Definition die umfassende Doktrin der Strict Liability und ihre zahlreichen Variationen nicht ganz. ${ }^{76}$

Um die strikte Verantwortlichkeit in ihrer Vielseitigkeit erfassen zu können, ist es zielführender, von einem weiten Verständnis auszugehen und eine Strafbarkeit dann als Strict Liability zu bezeichnen, wenn die Erhebung des Schuldvorwurfs in Abweichung zum üblicherweise platzierten Vorwurf vorgenommen wird, das heißt zu demjenigen im Einklang mit dem Schuldprinzip als Strafbegründungs- und als Strafzumessungsprinzip. ${ }^{77}$ Das heißt ein Schuldvorwurf ist immer dann „strikt" oder eben „strikter", wenn von den regulären Anforderungen an die Strafbarkeit abgewichen wird. Dabei können sich diese Abweichungen vom Schuldgrundsatz entweder auf die Tathandlung bzw. das Unrecht selbst oder auf das Urteil über den Täter erstrecken, das heißt entsprechend die Schuldkategorie im engeren Sinne oder im weiteren Sinne betreffen. Darüber hinaus kann sich die „Strenge“ des Vorwurfs auch prozedural, das heißt im Sinne einer Strict Procedural Liability niederschlagen, bei welcher das Verfahren nicht geeignet ist, den Schuldvorwurf angemessen abzubilden (z.B. im Rahmen von im angelsächsischen Recht weit verbreiteten Beweislastumkehrungen oder fixen Straftarifen) ${ }^{78}$ Eine Strict Liability Offense ist also eine Bestimmung, deren Konstruktion es nicht vermag, in Einklang mit dem Schuldprinzip sicherzustellen, dass die verhängte Strafe

73 So z.B. bei Duff, in: Simester (Hrsg.), Appraising Strict Liability (2005), 125, 125; Simons, Journal of Criminal Law and Criminology 87 (1997), 1075, 1080.

74 So z.B. Husak, Canadian Journal of Law and Jurisprudence 8 (1995), 189, 195.

75 Siehe z.B. Ashworth/Horder, Principles of Criminal Law, 7. Aufl. (2013), 160 f.; Green (Fn. 72), 2; Jefferson, Criminal Law, 12. Aufl. (2015), 122; Keating u.a., Clarkson and Keating Criminal Law, 8. Aufl. (2014), 227.

76 Siehe zu diesen Variationen und verschiedenen Kategorisierungsversuchen ausführlich Simmler (Fn. 54), 311 ff.

77 Siehe zu diesem Verständnis Simmler (Fn. 54), 326 ff.

$78 \mathrm{Zu}$ dieser Strict Procedural Liability im Detail Simmler (Fn. 54), 388 ff.; Husak (Fn. 74), 199 f. 
nur einen schuldigen Täter trifft und ferner in angemessenem Verhältnis zu seiner Schuld steht, bei der also ansonsten gerade zu diesem Zweck geschaffene Regelungen der Strafrechtsdoktrin missachtet werden.

\section{Anwendungsbereiche der strikten Verantwortlichkeit}

Oftmals wird, wie bereits erwähnt, behauptet, bei den Strict Liability Offenses handle es sich ausschließlich um Delikte zum Schutze des „öffentlichen Wohls“. Diese Begrifflichkeit schlägt jedoch unter anderem aufgrund der Weite der tatsächlichen Anwendungsbereiche der Doktrin fehl. Da die Strict Liability das US-amerikanische Recht so weitläufig erfasst, ist eine einfache Fallgruppeneinteilung kaum möglich. ${ }^{79}$ In der Literatur werden regelmäßig primär Beispiele aus dem Bereich der Straßenverkehrsdelikte, aus dem Bereich ebendieser sog. Public Welfare Offenses darunter zum Beispiel der Verkauf verdorbener Nahrungsmittel - und aus dem Bereich der Sittlichkeits- und Sexualdelikte im Zusammenhang mit Minderjährigen angeführt. ${ }^{80}$ In der Tat lassen sich summarisch zwei praxisrelevante Anwendungsbereiche anführen: Einerseits die Regulation des Wirtschaftslebens, des Verkehrs und anderer öffentlicher Aktivitäten im weitesten Sinne. ${ }^{81}$ Hier ist oftmals kein voller Nachweis des subjektiven Tatbestands erforderlich. ${ }^{82}$ Zudem stehen in diesem Bereich häufig auch juristische Personen im Fokus der Strafverfolgung. ${ }^{83}$ Ferner werden die Lockerungen des Schuldprinzips bei diesen Delikten oft damit legitimiert, dass diese Straftaten vergleichsweise niedrige Strafen vorsehen und kein besonderes Stigma nach sich zögen. ${ }^{84}$ Diese „Ideologie der Trivialität“ ${ }^{45}$, sollte man ihr denn überhaupt eine Berechtigung einräumen, kann jedoch keinesfalls für den zweiten, gerade in den USA relevanten Bereich der Strict Liability gelten: denjenigen der Sexual- und Tötungsdelikte. Diese

79 Dazu auch Safferling (Fn. 56), 375 f.

80 So auch Husak (Fn. 74), 192.

81 Vgl. B. Sullivan, in: Reed/Bohlander (Hrsg.), General Defences in Criminal Law (2015), 25, 29; Keating u.a. (Fn. 75), 227.

82 Norrie (Fn. 62), 83; Duff u.a., in: Duff u.a. (Hrsg.), Criminalization (Fn. 3), 3.

83 G.R. Sullivan, in: Simester (Hrsg.), Appraising Strict Liability (2015), 195, 196; Keating u.a. (Fn. 75), 227.

84 So der Supreme Court in Morissette v. United States, 342 U.S. 246, 256 (1952); dazu auch Greenberg/Brotman (Fn. 65), 80.

85 Kritisch zu dieser Ideologie Norrie (Fn. 62), 83 f., der anführt, dass gerade auch Verstöße des Wirtschaftsstrafrechts keineswegs weniger schwerwiegend seien; ähnlich auch Jefferson (Fn. 75), 123. 
Delikte des Kernstrafrechts mit erheblichen Sanktionsandrohungen spielen keineswegs eine unbedeutende Rolle, was jedoch oft vernachlässigt wird. $^{86}$

\section{E. Overcriminalization oder Overpunishment?}

\section{Strikte Verantwortlichkeit als Ausweitung der Kriminalisierung}

Wie bereits die summarisch aufgeführten Anwendungsbereiche offenbaren, handelt es sich bei der Strict Liability - entgegen der Vermutung, welche die aktuellen politischen Debatten nahelegen - keineswegs um ein Phänomen, welches einzig das Wirtschaftsstrafrecht erfasst. So ist für die Frage, ob sich ein direkter Zusammenhang zwischen „Überkriminalisierung" und Strict Liability ausmachen lässt, auch klar zwischen den verschiedenen Variationen eines strikten Schuldvorwurfs zu differenzieren.

Die im Rahmen der Mens Rea Debate vorgebrachte Kritik betrifft Delikte im Bereich des sog. Regulatory Law, bei denen sich die "Strenge“ des Schuldvorwurfs in der Regel dadurch charakterisieren lässt, dass dem Beschuldigten der subjektive Tatbestand nicht oder nicht vollumfänglich nachgewiesen werden muss. In diesem Zusammenhang kann durchaus davon gesprochen werden, dass die Strafbarkeit an sich ,ausgeweitet“" wird, indem Verhaltensweisen, die bei strenger Befolgung des Schuldprinzips strafrechtlich nicht erfasst würden, pönalisiert und damit verfolgbar werden. Entsprechend ist die Kritik in der Tat berechtigt, dass mit dieser Art von Deliktskonstruktionen die Grenzen des Strafrechts bzw. des strafrechtlich relevanten Bereichs erweitert werden und diese Debatte deshalb das Problem der Overcriminalization im engeren Sinne betrifft. Dies bezieht sich jedoch zunächst einmal nur auf die formale Kriminalisierung, und die Frage verbleibt - wenn man die Anwendungsbereiche dieser Delikte im Blick hat -, ob die hier im Fokus stehenden Delikte tatsächlich auch Grund für eine faktische Expansion von Strafverfolgungen und Inhaftierungen sind.

Gemäß Larkin beträfen mehr als $90 \%$ der Strafverfolgungen auf Bundesebene Straftaten, die nicht Gegenstand der Mens-Rea-Debatte seien. ${ }^{87}$

86 So auch Keating u.a. (Fn. 75), 227.

87 Larkin (Fn. 7), 2. 
Diese Schätzung ist plausibel. Betrachtet man die Inhaftierungsgründe, ${ }^{88}$ sind das Wirtschaftsstrafrecht bzw. eben diese Public Welfare Offenses für die Gefängnispopulation kaum von Relevanz, sind die Strafandrohungen in diesem Bereich doch selten hoch. Vielmehr sind es andere Delikte, die im Verdacht stehen, tastsächlich zu dieser faktischen Expansion des Strafrechts beizutragen, wie zum Beispiel die Strict Liability im Bereich von Sexual- oder Tötungsdelikten oder Lockerungen des Schuldprinzips im Bereich der eigentlichen Strafzumessung. Die von den Republikanern vorgeschlagene Mens Rea Reform beträfe allerdings explizit nicht solche Delikte, wie immer wieder betont wurde. ${ }^{89}$ Es stellt sich demnach die Frage, ob der politische Angriff auf die strikte Verantwortlichkeit wirklich den Kern der Doktrin bzw. des eigentlichen Problems trifft.

\section{Strikte Verantwortlichkeit als Überbestrafung}

Es ist die gängige These, dass die Ursache für die Masseninhaftierung in der „Überkriminalisierung“ liegt. ${ }^{90}$ Allerdings legen die bisherigen Ausführungen bereits nahe, dass diese Terminologie nur zu einem gewissen Grad passend ist, sind doch für die Inhaftierungsraten nicht primär die formale Ausweitung der Strafgesetzgebung, sondern vielmehr die tatsächlich verhängten Strafen und deren Ausmaß entscheidend. So hat der „Niedergang des Rehabilitationsideals“ in den letzten zwei Jahrzehnten zu einem massiven Anstieg von Strafen und Strafmaßen geführt, wobei gerade dem harten Umgang mit Drogendelinquenten und Wiederholungstätern Vorrang vor anderen - strafrechtstheoretischen und empirischen - Überlegungen eingeräumt wurde. ${ }^{91}$ Demzufolge drängt sich der Verdacht auf, dass das, was gemeinhin als Overcriminalization thematisiert wird, eigentlich gar nicht die Frage nach den Grenzen des Strafrechts selbst betrifft,

88 Genaue Zahlen, wie viele Personen aufgrund einer Public Welfare Offense inhaftiert sind, liegen zwar nicht vor; 2015 waren jedoch in den Gefängnissen wegen Delikten unter der Jurisdiktion der Bundesstaaten 52,9\% der Insassen wegen eines Gewaltdelikts, $19 \%$ wegen eines Eigentumsdelikts, $15,7 \%$ wegen eines Drogendelikts und $11,6 \%$ wegen eines Delikts gegen die öffentliche Ordnung inhaftiert. Nur $0,8 \%$ waren wegen ,anderem“ im Gefängnis. Diese Zahlen deuten bereits an, dass die Delikte, welche im Zentrum der Mens Rea Debate stehen, kaum für die Masseninhaftierung verantwortlich gemacht werden können; siehe U.S. Department of Justice, Bureau of Justice Statistics, Prisoners in 2015.

89 Siehe dazu z.B. Yaffe (Fn. 51).

90 Siehe z.B. Koch/Holden (Fn. 4).

91 So auch Luna (Fn. 20), 710. 
sondern zu einem deutlich größeren Teil ein Problem einer „Überbestrafung" darstellt. In der Tat sind ein solches Overpunishment und die bereits aufgeführten Problemkreise durchaus zu Recht auch Gegenstand der Overcriminalization-Debatte und entsprechend nicht nur die klassischen Strict Liability Offenses im Bereich des Wirtschaftsstrafrechts, sondern vielmehr auch andere Eingriffe in die allgemeine Geltung beanspruchende Verbrechenslehre. ${ }^{92}$

Betrachtet man die anderen Variationen der Strict Liability, offenbart sich schnell, dass diese mindestens so viel Aufmerksamkeit im Gezerre um die Strafrechtsreform verdienen. Während die Fragen, welche Verhaltensweisen überhaupt vom Strafrechtssystem erfasst sein sollen und inwiefern die Strict Liability dessen Anwendungsbereich ausweitet, die „Überkriminalisierung" im engeren Sinne betreffen, geht es bei den Lockerungen des Schulprinzips im Kernstrafrecht regelmäßig um Fragen einer eigentlichen „Überbestrafung“. Diese Formen strikter Verantwortlichkeit betreffen sodann primär das Schuldprinzip im Sinne des Proportionalitätsprinzips. Als Kernbestand der modernen Strafrechtstradition enthält der Schuldgrundsatz zwei Implikationen: Im Allgemeinen hält das Schuldprinzip fest, dass Strafe den Einzelnen nur treffen darf, wenn ihm ein persönlicher Vorwurf gemacht werden kann. ${ }^{93}$ Neben der Strafbegründungsschuld entfaltet das Schuldprinzip jedoch auch Wirkung hinsichtlich der Strafzumessungsschuld. Im Sinne des Verhältnismäßigkeitsprinzips impliziert das Schuldprinzip also des Weiteren auch, dass die Sanktion in einem angemessenen Verhältnis zur Schwere der Schuld stehen muss. ${ }^{94}$ Strict Liability Offenses sind nun oftmals gerade in Bezug auf dieses zweite Kriterium „strikt“ in dem Sinne, dass den Täter zwar sehr wohl eine Schuld trifft, die rechtlich vorgesehene Strafe jedoch nicht in einem angemessenen Verhältnis zur Schuld steht bzw. sonst relevante Kriterien für die Feststellung des Maßes der Schuld keine Berücksichtigung finden.

Die Kritik an der strikten Verantwortlichkeit bezieht sich demzufolge auch nicht nur auf die Strict Liability im Bereich des Nebenstrafrechts, sondern betrifft auch andere umstrittene Konstruktionen. Zu nennen wären hier die Delikte, bei denen Tatbestandsirrtümer als irrelevant gelten, wie bei der sog. Statutory Rape, das heißt bei der Vornahme sexueller Hand-

92 Siehe z.B. Luna (Fn. 20), 715 f.

93 Anstatt vieler z.B. Fuchs, Strafrecht AT I, 9. Aufl. (2016), Kap. 2 Rn. 24.

94 Siehe so z.B. Tully (Fn. 55), 24. 
lungen mit Minderjährigen, oder auch bei verschiedenen Besitzdelikten. ${ }^{95}$ Ferner verdient gerade auch die sog. Constructive Liability Aufmerksamkeit, bei welcher das Deckungsprinzip missachtet wird, das heißt bei welchen sich in der Regel der subjektive Tatbestand nicht auf den tatbestandsmäßigen Erfolg beziehen muss. ${ }^{96}$ Im Sinne des kanonischen Prinzips versari in re illicita werden in diesen Fällen demjenigen, der etwas Verbotenes tut, alle daraus resultierenden Folgen zugerechnet. ${ }^{97}$ Bei der Constructive Liability wird demnach ein weniger schwerwiegendes Delikt in ein schwereres transformiert, obwohl der Erfolg nicht vom Mens Rea umfasst ist. Entsprechend handelt es sich hier um eine strikte Verantwortlichkeit in Bezug auf den Taterfolg. ${ }^{98}$ Bekannteste und aufgrund sehr hoher Strafandrohungen weitreichendste Beispiele solcher Konstruktionen sind die sog. Felony Murder Rule und die Misdemeanor-Manslaughter Rule, das heißt Vergehen oder Verbrechen mit Todesfolge. ${ }^{99}$ Im Vergleich zu den im deutschsprachigen Raum als „erfolgsqualifizierte Delikte“ bezeichneten Konstruktionen, bei denen der Erfolg zumindest fahrlässig herbeigeführt werden muss, wird in den USA die innere Tatseite in Bezug auf den qualifizierenden Erfolg regelmäßig gänzlich außer Acht gelassen. ${ }^{100}$

Neben diesen Beispielen strikter Strafbarkeit, bei denen Lockerungen des Schuldprinzips zu deutlich höheren Strafmaßen und ferner auch zu einer verschärften und unverhältnismäßigen Stigmatisierung führen, sind des Weiteren auch die bereits angesprochenen Problemfelder der Strafzumessung im engeren Sinne anzusprechen. Diese Variation der strikten Verantwortlichkeit betrifft Bestimmungen, bei welchen die konkrete Strafzumessung selbst nicht mehr proportional zum Verschulden des Täters ist. Neben den Mandatory Minimums und anderen fixen Straftarifen, bei denen Richterinnen und Richter in ihrer Strafzumessung oftmals maßgeblich eingeschränkt werden, findet sich das eklatanteste Beispiel solcher

95 Siehe dazu z.B. Simons (Fn. 73), 1075 ff.; Weik (Fn. 58), 266 ff.; Simmler (Fn. 54), $361 \mathrm{ff}$.

96 Ashworth (Fn. 8), 411; Ashworth/Horder (Fn. 75), 75 ff.

97 Müller-Dietz, Grenzen des Schuldgedankens (1967), 15; Ashworth/Horder (Fn. 75), 76, Hörster (Fn. 55), 23.

98 So auch Duff (Fn. 73), 127.

99 Dazu z.B. Weik (Fn. 58), 275 ff.; Simmler (Fn. 54), 340 ff.

100 Weik (Fn. 58), 307 f. Die erfolgsqualifizierten Delikte unterscheiden sich entsprechend durchaus von der Constructive Liability; so auch Spencer/Pedain, in: Simester (Hrsg.), Appraising Strict Liability (2005), 237, 237; dazu auch Simmler (Fn. 54), 343 ff. 
Bestimmungen in der sog. „Three strikes and you are out“-Doktrin. ${ }^{101}$ Dieses „largest penal experiment in American history“"102 findet sich nach wie vor in zahlreichen US-amerikanischen Rechtsordnungen und führt zu einem besonders harten Umgang mit Wiederholungstätern, bei welchen die Schuld in Bezug auf das als drittes verübte Delikt keine Relevanz mehr hat. Für diese dritte Tat drohen je nach konkreter Ausgestaltung der Regelung in den Bundesstaaten 25 Jahre Haft oder sogar eine lebenslange Freiheitsstrafe (oft ohne Bewährung). ${ }^{103}$

Auch wenn sich zweifellos noch dutzende weitere Beispiele relevanter Einschnitte in das Schuldprinzip finden ließen, zeigt bereits dieser summarische Abriss, dass es sich bei der Kritik der Expansion des Strafrechts nur $\mathrm{zu}$ einem gewissen Grad um eine Frage der Kriminalisierung handelt. Vielmehr stehen nicht nur Fragen der Grenzen des Strafrechts, sondern auch dessen Funktionsweise im Zentrum. Es ist deshalb nicht gerechtfertigt, dass die Mens Rea Debate diese Formen der strikten Verantwortlichkeit im Sinne einer illegitimen Überbestrafung nicht miteinbezieht. Hier zeigt sich einerseits eine gewisse Undifferenziertheit der Debatte. Zweifelsohne offenbaren sich hier andererseits aber auch politische Interessenlagen.

\section{Klientelpolitik und die Variationen strikter Verantwortlichkeit}

Es konnte in den vorherigen Abschnitten aufgezeigt werden, dass die Overcriminalization im weiteren Sinne sowohl Fragen der eigentlichen Kriminalisierung als auch solche, welche hier als Overpunishment bezeichnet wurden, berührt. Es konnte ferner aufgezeigt werden, dass die Frage des Mens-Rea-Erfordernisses bzw. der Einhaltung des Schuldprinzips nicht zu Unrecht Gegenstand der Diskussionen um die Overcriminalization ist, dass aber gleichzeitig das Schuldprinzip nicht nur im von den Republikanern thematisierten Bereich der Public Welfare Offenses, sondern zweifelsohne auch im Kernstrafrecht mehr Beachtung geschenkt werden sollte. Möchte man dem Schuldprinzip angemessen Rechnung tragen und der Doktrin der strikten Verantwortlichkeit Einhalt gebieten, um

$101 \mathrm{Zu}$ den Three Strikes Laws allgemein z.B. Shichor/Sechrest, Three Strikes and You're Out (1996); Zimring u.a., Punishment and Democracy. Three Strikes and You're Out in California (2001).

102 Zimring u.a. (Fn. 101), 17.

103 Siehe z.B. California Penal Code, s. 667. 
der Verfolgung Unschuldiger, vor allem aber auch unverhältnismäßigen Strafverfolgungen, ein Ende zu setzen, müsste die Criminal Justice Reform auch die zahlreichen besprochenen Variationen der Strict Liability ins Auge fassen, welche es außerhalb des Wirtschaftsstrafrechts gibt.

Dass diese Lockerungen des Schuldprinzips zum Beispiel bei Sexualoder Tötungsdelikten nicht Gegenstand der Mens Rea Debate sind, sondern explizit ausgenommen sein sollen, lässt sich mit den politischen Interessenslagen und damit einhergehender Klientelpolitik erklären. Während die von den Republikanern scharf kritisierten Formen der Strict Liability einerseits primär Unternehmen oder Personen in Führungspositionen betreffen oder andererseits andere Personen, denen aufgrund einer speziellen Stellung Verantwortlichkeit zugeschrieben wird (zum Beispiel Hauseigentümer), trifft die strikte Verantwortlichkeit in anderen Bereichen, welche regelmäßig zu einer mit dem Schuldprinzip nicht zu vereinbarenden Überbestrafung führt, eher Straftäter mit „klassischem“ Profil, das heißt sozial Benachteiligte oder am Rande der Gesellschaft Stehende. Es überrascht wenig, dass sich die Verfechter der Mens Rea Reform - eben zum Beispiel die Gebrüder Koch oder Wirtschaftsverbände - weniger leidenschaftlich für eine Entkriminalisierung oder genauer gesagt Weniger-Kriminalisierung dieser Klientel einsetzt. Dies wäre jedoch für einen echten Wandel im Rahmen der Strafrechtsreform notwendig.

\section{F. Fazit: Das Schuldprinzip als Antwort auf Overcriminalization und Overpunishment}

Die vorangehenden Ausführungen haben demonstriert, dass sich die „Politik im Schuldprinzip“ gerade in der Debatte um die sog. Strict Liability und deren Relevanz für den Kampf gegen die Overcriminalization offenbart. Es sind diese Lockerungen der „Standardfunktionsweise“ des Strafrechts, das heißt der Orientierung sowohl am Grundsatz nulla poena sine culpa als auch an demjenigen nulla poena extra culpam, ${ }^{104}$ welche die Gemüter nicht nur aus strafrechtstheoretischer Perspektive bewegen. Sie erhalten insofern kriminalpolitische Relevanz, als diese Bestimmungen mitunter geeignet sind, die Strafrechtsgrenzen, das heißt die Grenzen strafrechtlicher Verantwortlichkeit, zu konstituieren. Darüber hinaus sind sie im Sinne des hier als Overpunishment bezeichneten Phänomens auch für den konkreten Umgang mit Straftätern relevant und führen regelmäßig zu

104 Vgl. Tully (Fn. 55), 24. 
einer disproportionalen Strafe, was die Problematik der Masseninhaftierung verschärft. Trotzdem kommt in der politischen Mens Rea Debate straftheoretischen und kriminalisierungstheoretischen Überlegungen ungleiches Gewicht zu, wobei die jeweilige Priorisierung maßgeblich politideologisch geprägt ist. Welche „Baustellen“ des Schuldprinzips, das heißt welche seiner Lockerungen zuerst oder ob sie überhaupt angegangen werden sollen, scheidet die politischen Geister und ist deshalb geeignet, den neuen kriminalpolitischen „Konsens“ hinsichtlich der Notwendigkeit von Reformmaßnahmen zu gefährden.

Es wird sich noch zeigen, in welche Richtung sich die Debatte entwickelt. Dass den Strict Liability Offenses weitreichend Einhalt geboten wird, ist dabei unwahrscheinlich. Dass jedoch die aktuelle Diskussion um die Erfordernisse an die innere Tatseite zumindest das Potenzial hat, mehr politische Sensibilität für ein in der Strafrechtswissenschaft lange thematisiertes und kritisiertes Phänomen zu schaffen, bleibt zu hoffen. Die konsequente Befolgung des Schuldprinzips wäre, wie aufgezeigt werden konnte, durchaus geeignet, einen wesentlichen Beitrag zur Entschärfung des USamerikanischen „Strafrechtsproblems“ zu leisten. Für den Bestand des Schuldprinzips im Strafrecht der USA - und anderswo - zeigt dieser Themenkomplex deutlich, dass die Eingriffe der Politik (und gerade der Klientelpolitik) in die eigentlich vorgesehene Funktionsweise des Strafrechts unter anderem für die Instabilität des US-amerikanischen Strafrechts (mit-)verantwortlich erklärt werden können und damit die Aufgabenerfüllung des Strafrechts beeinträchtigen. Es wäre in diesem Sinne zu befürworten, dass die Politik, wenn sie das Strafrecht zum Schutze bestimmter Normen in Anspruch nimmt, auch dessen Funktionsweise respektiert. Dies bedeutete weder, die Strafbarkeit auf Delikte auszuweiten, für welche keine innere Tatseite nachgewiesen werden muss (in der Tat eine Overcriminalization), noch Strafen zu verhängen, welche nicht in einem angemessenen Verhältnis zur Schuld des Täters stehen (in der Tat ein Overpunishment). Oder kurz: das Schuldprinzip einzuhalten. 